\title{
Atuação de fisioterapeutas na inclusão de alunos com deficiência física no ensino regular
}

\section{Resumo}

Francisco Ricardo Lins Vieira de Melo Universidade Federal do Rio Grande do Norte

Neide Maria Gomes de Lucena

Universidade Federal da Paraíba

Luzia Lívia Oliveira Saraiva

Universidade Federal do Rio Grande do Norte

O estudo teve como objetivo identificar a atuação do fisioterapeuta no processo de inclusão escolar de alunos com deficiência física. Trata-se de uma pesquisa transversal, do tipo descritiva com abordagem quantitativa. Por meio de um questionário, participaram 47 fisioterapeutas, atuantes na área de neuropediatria de cinco centros de reabilitação da cidade de João Pessoa, na Paraíba. Os resultados mostraram que o contexto escolar dos alunos com deficiência física é um campo a ser mais bem explorado pelos fisioterapeutas. Para tanto, é necessária uma formação acadêmica mais condizente com o avanço das concepções acerca da deficiência e das políticas educacionais numa perspectiva multiprofissional, tendo como foco uma maior participação e aprendizagem das pessoas com deficiência física no ensino regular.

Palavras-chave: Inclusão escolar. Deficiência física. Fisioterapia.

\section{The role of physiotherapists in the inclusion of students with physical disabilities into regular education}

\section{Abstract}

The study had the goal of identifying the physiotherapist's performance in the school inclusion process for students with physical disabilities. It is a descriptive, cross-sectional research, with a quantitative approach. Forty seven physiotherapists who work in neuropediatrics in five rehabilitation centers in the city of João Pessoa, Paraíba took part in the survey. The results showed that the school context for students with physical disabilities is a field to be better explored by physical therapists. In order to do so, it is necessary to have an academic education more in line with the advancement of conceptions about disability and educational policies in a multiprofessional perspective, focusing on a greater participation and learning of people with physical disabilities in regular education. Keywords: School inclusion. Physical disabilities. Physiotherapy. 


\section{Actuación de fisioterapeutas en la inclusión de alumnos con dis- capacidad física en la enseñanza regular}

\section{Resumen}

El estudio objetivó identificar la actuación del fisioterapeuta en el proceso de inclusión escolar de alumnos con discapacidad física. Se trata de una investigación transversal, del tipo descriptivo con abordaje cuantitativo. La recolección de datos se realizó por medio de un cuestionario, con la participación de 47 fisioterapeutas actuantes en el área de neuropediatría de cinco centros de rehabilitación de la ciudad de João Pessoa en Paraíba. Los resultados han mostrado que el contexto escolar de los alumnos con discapacidad física es un campo a ser mejor explorado por los fisioterapeutas. Para tanto, es necesaria una formación académica más relacionada con el avanzo de las concepciones acerca de la discapacidad y de las políticas educacionales en una perspectiva multiprofesional, con foco en una mayor participación y aprendizaje de las personas con discapacidad física en la enseñanza regular.

Palabras clave: Inclusión escolar. Discapacidad física. Fisioterapia.

\section{Introdução}

A inclusão escolar de alunos com deficiência no sistema regular de ensino tem sido extensivamente abordada em pesquisas, tanto da área de educação como de saúde. No Brasil, ela é um direito garantido pela Lei de Diretrizes e Bases da Educação Nacional, Lei n. 9.394, de 20 de dezembro de 1996.

Entre os diversos dispositivos que objetivam a consolidação da política educacional inclusiva, destaca-se a Política Nacional de Educação Especial na Perspectiva da Educação Inclusiva, em vigor desde 2008. Essa política definiu a Educação Especial como modalidade transversal ao sistema de ensino regular e redimensionou a população atendida por esse sistema, elegendo, como público-alvo da Educação Especial, os estudantes com deficiência, transtornos globais do desenvolvimento e altas habilidades/superdotação (BRASIL, 1996; 2008a).

Visando assegurar a efetiva inclusão escolar desses alunos, a Política Nacional de Educação Especial na Perspectiva da Educação Inclusiva garante, também, a disponibilização de serviços e recursos próprios para o 
Atendimento Educacional Especializado durante a trajetória dos educandos no ensino regular (BRASIL, 2008a).

Em se tratando das pessoas com deficiência física-definida como variedade de condições não sensoriais que afetam o indivíduo em termos de mobilidade, coordenação motora geral ou da fala, em decorrência de lesões neurológicas, neuromusculares e ortopédicas, ou ainda, malformações congênitas ou adquiridas - sabe-se que os comprometimentos motores que acompanham esses indivíduos podem estar interferindo na participação deles no contexto escolar (BRASIL, 2002). Além da condição da deficiência, muitas vezes, essa participação é restrita por condições impostas pela sociedade através de barreiras arquitetônicas, atitudinais, de comunicação, entre outras (MELO, 2006).

Sendo assim, os alunos com deficiência física carecem de modificações no ambiente escolar que podem envolver a adequação do espaço físico, de material escolar, de recursos pedagógicos, equipamentos e mobiliário escolar específico, bem como de recursos humanos capacitados para atuar junto a esse alunado, possibilitando, assim, oportunidades iguais e um desempenho satisfatório nas atividades desenvolvidas nesse meio (SARAIVA; MELO, 20111 .

Considerando que as dificuldades ou limitações apresentadas pelos alunos com deficiência física são específicas e produzem necessidades de avaliação e intervenção diferenciadas, a atuação de uma equipe multiprofissional que inclua profissionais das mais diversas áreas (humanas, saúde, tecnológica) é de fundamental importância para o sucesso das ações que visam otimizar a aprendizagem desses estudantes (FERREIRA, 2007; OLIVEIRA; MELO; ELALI, 2008).

É neste contexto que o fisioterapeuta, enquanto profissional da saúde, pode contribuir com a família, os professores e demais profissionais da equipe escolar para a construção de um ambiente que respeite e responda à diversidade, seja fornecendo subsídios para melhor lidar com os déficits motores do aluno com deficiência física, seja auxiliando no combate das barreiras atitudinais, entre outras, que interferem na participação e plena inclusão.

Entretanto, no Brasil, o contexto educacional ainda é pouco explorado como campo de atuação do fisioterapeuta, especialmente quando é voltada para o aluno com deficiência física no âmbito das escolas regulares, como 
Francisco Ricardo Lins Vieira de Melo| Neide Maria Gomes de Lucena | Luzia Lívia Oliveira Saraiva

mostram algumas pesquisas (TAGLIARI; TRÊS; OLIVEIRA, 2006; MARTINS; MELO, 2010 ; SARAIVA; MELO, 2011 ; NIEHUES; NIEHUES, 2014).

É possível que tal constatação esteja relacionada com o processo de inclusão relativamente recente no nosso país, além de uma formação acadêmica que, em muitos casos, ainda se mantém centrada no modelo médico, em detrimento do modelo social da deficiência.

$\bigcirc$ que diferencia o "modelo médico da deficiência" do "modelo social de deficiência" é a forma como se concebe o entendimento acerca da condição da deficiência.

Na perspectiva do modelo médico, a deficiência é vista como um problema exclusivo da pessoa; , por essa razão, precisa ser "corrigida" (melhorada, curada, entre outros), a fim de que possa fazer parte da sociedade.

Já na perspectiva do modelo social, a deficiência não deve ser entendida como dificuldade do indivíduo, mas como problemática inerente à sociedade como um todo, visto que as limitações e dificuldades vivenciadas pela pessoa com deficiência decorrem das barreiras (arquitetônicas, comunicacionais, transporte, tecnológicas, atitudinais, etc.) que a sociedade impõe, impedindo o pleno desenvolvimento e inserção nos diversos contextos sociais - educacional, laboral, lazer, saúde, entre outros (SASSAKI, 2005).

No entanto, vale salientar que, com base na proposição do modelo da Classificação Internacional de Funcionalidade, Incapacidade e Saúde (CIF) pela Organização Mundial de Saúde (OMS) no ano de 2001 e revisões posteriores, houve um avanço no entendimento acerca da concepção da deficiência, sendo gradualmente incorporada em diversos setores, como saúde e educação, além de equipes multidisciplinares (ORGANIZAÇÃO MUNDIAL DE SAÚDE, 2004).

Segundo a CIF (Figura 1), a deficiência surge da interação entre problemas de saúde e fatores contextuais (fatores ambientais e pessoais), capazes de influenciar a funcionalidade humana. Os fatores ambientais incluem: produtos e tecnologias, o ambiente natural e o construído, suporte e relacionamentos, atitudes, serviços, sistemas e políticas públicas. Esses fatores podem alterar os níveis de funcionalidade e incapacidade, atuando como facilitadores ou barreiras que auxiliam ou limitam a execução de certas atividades e a participação da pessoa com deficiência (ORGANIZAÇÃO MUNDIAL DE SAÚDE, 2004). 


\section{Figura 1}

\section{Interação entre os componentes da ClF}

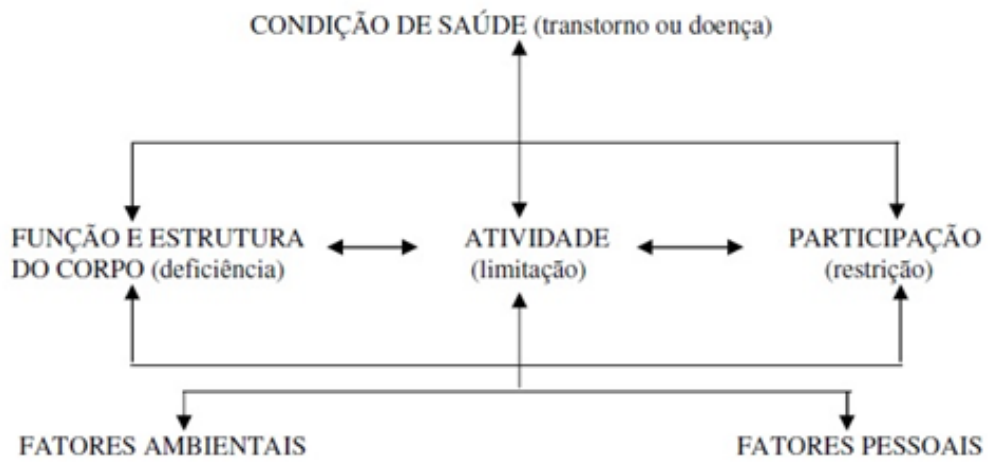

Fonte | Adaptação de Araújo (2008).

Dessa forma, os ambientes físico, social e comportamental podem "incapacitar" as pessoas com deficiência ou, por outro lado, fomentar sua participação e inclusão. A deficiência passa, então, a ser entendida como um

180 "modelo biológico-psíquicosocial". Ratificado pelo Relatório Mundial sobre a Deficiência, publicada em 2011 , esse modelo representa um compromisso viável entre os modelos médico e social da deficiência, reconhecendo que a deficiência não pode ser definida exclusivamente com base em barreiras físicas ou sociais (ORGANIZAÇÃO MUNDIAL DE SAÚDE, 2012).

Sendo a fisioterapia a profissão da área da saúde que se relaciona diretamente com a funcionalidade e a incapacidade, tendo em vista que a função e o movimento são objetos de estudo, espera-se que essa área considere e se embase no modelo da CIF ao trabalhar com a recuperação funcional, prevenção de incapacidades e com a qualidade de vida dos indivíduos (ARAÚJO, 2008).

De fato, alguns autores apontam que tem ocorrido uma mudança filosófica das intervenções fisioterapêuticas que ultrapassam a proposta de "correção" da deficiência física subjacente aos problemas funcionais, mas passam a ter um enfoque adicional no ambiente dessas pessoas, maximizando a sua independência em atividades diárias e sua participação na comunidade, inclusive na escola (NOVAK; MCINTYRE; MORGAN; CAMPBELL; DARK; MORTON; STUMBLES; WILSON; GOLDSMITH, 20131. 
Por entender que o fisioterapeuta pode e necessita atuar no sentido de mudar as estruturas e atitudes da sociedade para incluir, em seu seio, as pessoas com deficiência física, oportunizando o seu desenvolvimento pessoal, educacional, profissional e social, é que surge a seguinte questão norteadora deste estudo: os fisioterapeutas que atendem a crianças e adolescentes com deficiência física estão atuando no seu processo de inclusão escolar?

Como objetivo, procurou-se: identificar a atuação do fisioterapeuta no processo de inclusão escolar de alunos com deficiência física, na cidade de João Pessoa, Paraíba.

\section{Metodologia}

Trata-se de um estudo transversal, do tipo descritivo com abordagem quantitativa (GIL, 2010).

projeto de pesquisa foi encaminhado e aprovado, anteriormente à sua execução, pelo Comitê de Ética em Pesquisa em Seres Humanos da Universidade Federal da Paraíba, sendo favorável o Parecer n 332/1 1 .

Para levar a efeito o estudo, foram identificadas as instituições na cidade de João Pessoa que realizavam atendimento fisioterapêutico em neuropediatria, através do registro de empresa no Conselho Regional de Fisioterapia e Terapia Regional da primeira região (CREFITO 1). Em seguida, foram efetivadas visitas aos centros de reabilitação visando fornecer esclarecimentos e obter ou não a anuência para participação no estudo, sendo alcançada a concordância de todas as instituições.

Ademais, todos os fisioterapeutas foram convidados, pessoalmente, a participar voluntariamente na condição de informante da pesquisa, sendo unânime a assinatura do Termo de Consentimento Livre e Esclarecido.

Foi organizado um cronograma e, em horário previamente estabelecido com os sujeitos da pesquisa, os pesquisadores compareceram aos serviços de reabilitação para aplicar o questionário presencialmente, sendo a coleta de dados realizada no período de janeiro a março de 2012.

Participaram da pesquisa 47 fisioterapeutas atuantes na área de neuropediatria de cinco centros de reabilitação (públicos e privados) da cidade de João Pessoa, Paraíba. 
questionário utilizado como instrumento de coleta de dados foi constituído de perguntas que versavam sobre o tempo de formação profissional, atuação no processo de inclusão escolar de crianças e adolescentes com deficiência física, preparo para atuarem no processo de inclusão escolar e se consideravam efetivo o trabalho de uma equipe multidisciplinar na perspectiva da inclusão dos alunos com deficiência física na escola regular.

Após a coleta, os dados foram organizados e submetidos à análise quantitativa por meio da análise estatística descritiva, utilizando-se o programa EXCEL. Os dados foram representados por gráficos visando favorecer a discussão dos resultados da pesquisa.

\section{Resultados e discussão}

Participaram do estudo 47 fisioterapeutas com tempo médio de formação acadêmica de 9, 1 anos.

Questionados sobre a sua participação no processo de inclusão escolar de crianças e adolescentes com deficiência física, 19 (40,4\%) disseram que atuam, enquanto 28 (59,6\%) não, conforme Gráfico

\section{Gráfico 1}

Participação dos fisioterapeutas no contexto escolar de crianças e adolescentes com deficiência física

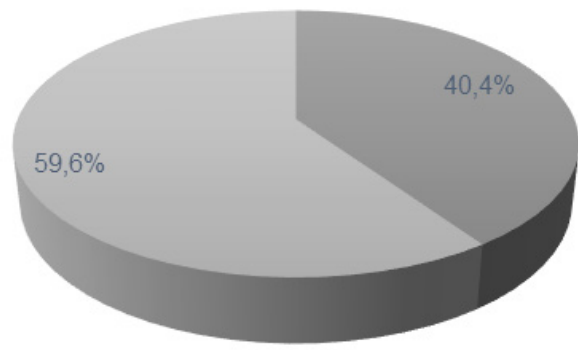

- Sim — Não

Fonte | Dados da pesquisa.

desconhecimento por parte dos professores a respeito do diagnóstico clínico e funcional de alunos com deficiência física pode implicar 
dificuldades na identificação das limitações e do potencial desse público (ALPINO, 2008; LANDMANN; RUZZA; CHESANI; 2009).

São recorrentes as pesquisas que indicam demandas relacionadas aos alunos com deficiência física que carecem de contribuições do fisioterapeuta como manuseio, locomoção, posicionamento, adaptações de recursos e materiais (como material pedagógico, mobiliário), bem como de atividades motoras e brincadeiras no ambiente escolar (ALPINO, 2008; BRACCIALLI; MANZINI; REGANHAN, 2004; DURCE; PEREIRA; FERREIRA, SOUZA, 2006; ALPINO; SILVA; KARIATSUMARI; SOUZA; OKURO; ARAÚJO, 2007; JORQUEIRA NETO; BLASCOVIASSIS, 2009; SARAIVA; MELO, 2011 ; MORINA, 2013; SANTOS; LARA; FOLMER, 2015).

Nesse sentido, cita-se, como apoio essencial ao melhor desempenho funcional e escolar do aluno com deficiência física e, certamente, no que diz respeito à maior segurança dos professores, a orientação profissional especializada quanto à adaptação da mobília, materiais e recursos que possam favorecer a boa postura, mobilidade, comunicação e utilização das mãos. Dessa forma, os professores receberiam suporte adequado diante dos aspectos que parecem constituir os maiores obstáculos ao atendimento educacional desse alunado (ALPINO, 2008).

Em se tratando das crianças com deficiência física decorrente de lesão neurológica, os cuidados a serem tomados no âmbito das escolas podem se tornar ainda mais complexos, tendo em vista que, além dos transtornos de controle do movimento e da postura, outros fatores poderão interferir diretamente na independência e autonomia desses alunos, como as complicações vesicais, intestinais, musculoesqueléticas, entre outras (MELO; FERREIRA; 2009).

Ademais, a deficiência física pode vir associada a outros tipos de deficiência (visual, auditiva, intelectual), ao autismo, entre outras condições, que carecem que o professor obtenha informações dessas outras áreas, a fim de promover um adequado atendimento do aluno no contexto escolar (MELO; FERREIRA; 2009).

Para tanto, além da conversa entre professores e a família do aluno com o objetivo de conhecer e lidar com essas particularidades, a atuação de profissionais de saúde, como o fisioterapeuta e o terapeuta ocupacional mostra-se relevante. Cabe a esses profissionais, em seu trabalho com a equipe escolar, observar e buscar soluções que auxiliem os alunos com deficiência 
Atuação de fisioterapeutas na inclusão de alunos com deficiência física no ensino regular

física nos aspectos relacionados às questões posturais, de locomoção e de adequação do mobiliário escolar, entre outros, facilitando o trabalho pedagógico (MELO; FERREIRA, 2009; SARAIVA; MELO, 2011 ).

Além disso, outros estudos citam que o fisioterapeuta pode atuar nas orientações quanto à acessibilidade física da escola, tendo em vista esta ser um dos primeiros requisitos que possibilitam aos alunos o acesso e circulação pelas dependências escolares, permitindo-lhes utilizar funcionalmente todos os espaços (ARANHA, 2004; ALPINO; SILVA; KARIATSUMARI; SOUZA; OKURO; ARAÚJO., 2007; MORINA, 2013; GALLO; ORSO; FIÓRIO, 20111.

Os fisioterapeutas também podem contribuir para o combate de preconceitos, medos e estigmas formados sobre pessoas com deficiência física, considerando que as barreiras atitudinais as isolam e/ou dificultam sua inclusão social (ARNS, 2006; MORINA, 2013).

Apesar de conhecida a importância da atuação do fisioterapeuta no contexto escolar, a maioria dos profissionais do presente estudo não participam das ações voltadas para melhorar o aprendizado e a inclusão dos alunos com deficiência física, embora lidem diretamente com crianças e adolescentes com essas condições em sua prática clínica. Essa pouca participação também foi apontada em outros trabalhos (TAGLIARI; TRÊS; OLIVEIRA, 2006; JORQUEIRA NETO, BLASCOVI-ASSIS, 2009; SARAIVA; MELO, 201 1; SANTOS; LARA; FOLMER, 2015).

Autores, como Silva e Mazzota (2009), Melo e Pereira (2013), sinalizam a necessidade de mais visitações de fisioterapeutas às escolas de crianças com deficiência física, seja o graduando ou o profissional do serviço de reabilitação, bem como de um melhor preparo, através de estudos e ações que favoreçam a inclusão de pessoas com deficiência nos vários setores da sociedade, destacadamente no contexto escolar.

Quanto aos estudos voltados para a atuação do fisioterapeuta na escola, constata-se que a literatura é bastante escassa, principalmente, quando se trata do aluno com deficiência física (SARAIVA; MELO, 2011 ; NIEHUES; NIEHUES, 2014; SANTOS; LARA; FOLMER, 2015).

A pesquisa de Martins e Melo (2010) realizou uma análise de dissertações e teses brasileiras que teve, como temática, a interface entre a fisioterapia e a educação inclusiva e encontrou, apenas, seis trabalhos publicados no 
período de 2002 a 2008. Com isso, evidenciou-se uma lacuna na produção de conhecimento na área da fisioterapia em interface com a inclusão escolar.

Já a investigação de Niehues e Niehues (2014) que buscou os artigos que relacionam essa interface publicados nas bases de dados SciELO, LILACS, MEDLINE, Pubmed e nas bases de universidades, encontrou, apenas, 11 trabalhos.

Acerca do preparo para atuarem no processo de inclusão escolar, 20 fisioterapeutas consideraram estar parcialmente preparados, enquanto 18 citaram estar preparados, e nove indicaram despreparo (Gráfico 2).

\section{Gráfico 2}

Preparação dos fisioterapeutas para atuarem no processo de inclusão escolar.

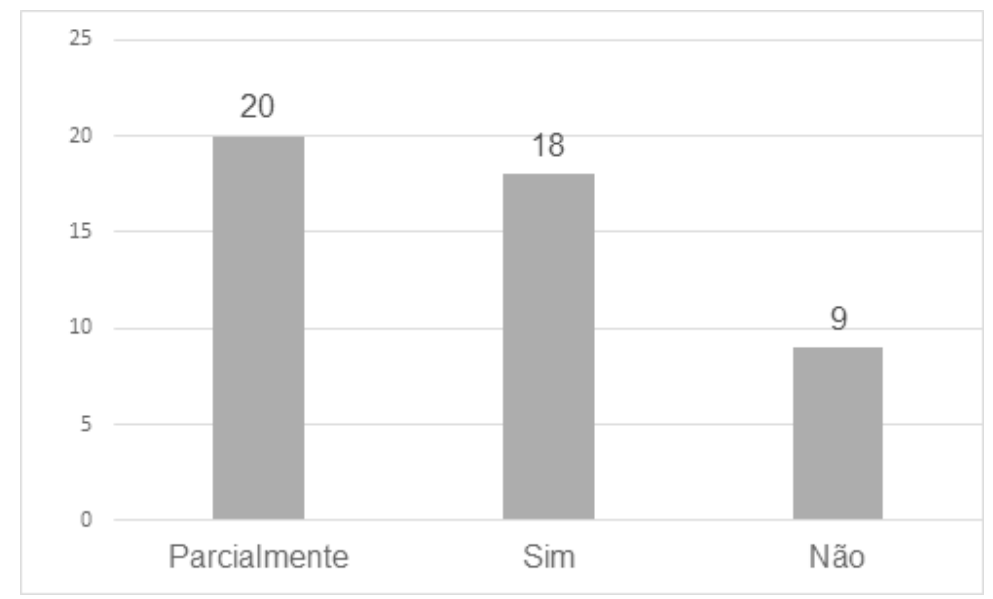

Fonte | Os autores.

Dos 47 participantes, o estudo mostrou que, apenas, oito cursaram alguma disciplina específica voltada ao atendimento educacional da pessoa com deficiência física em sua graduação e 35 dos profissionais disseram que não participaram de nenhuma formação continuada voltada para a temática.

Vale salientar que, por considerar necessário complementar os currículos de formação docente e de outros profissionais que lidam com as pessoas com deficiência, o Ministério da Educação (MEC), por meio da Portaria $n^{\circ}$ 1.793, de 27 de dezembro de 1994, recomenda a inclusão da disciplina "Aspectos ético-político-educacionais da normalização e integração da 
Atuação de fisioterapeutas na inclusão de alunos com deficiência física no ensino regular

pessoa portadora de necessidades especiais" prioritariamente, nos cursos de Pedagogia, Psicologia e em todas as Licenciaturas, e também conteúdos referentes a esses aspectos nos cursos do grupo de Ciências da Saúde (Educação Física, Enfermagem, Farmácia, Fisioterapia, Fonoaudiologia, Medicina, Nutrição, Odontologia, Terapia Ocupacional), no Curso de Serviço Social e nos demais cursos superiores, de acordo com as suas especificidades. A portaria ainda recomenda a manutenção e expansão de estudos adicionais, cursos de graduação e de especialização já organizados para as diversas áreas da Educação Especial (BRASIL, 1994).

Embora esses conteúdos devam estar presentes durante a graduação do fisioterapeuta, $39(82,9 \%)$ profissionais da presente pesquisa informaram não terem esse componente em suas disciplinas curriculares.

Também no trabalho de Mendes (2006), os professores e alunos do curso de fisioterapia de uma instituição de ensino superior do Paraná não obtiveram informações a respeito do assunto durante suas respectivas graduações, embora considerassem importante para um atendimento adequado de crianças com deficiência física.

A despeito de a maioria dos fisioterapeutas informarem que não adquiriram conhecimentos acerca da inclusão de pessoas com deficiência física no ensino formal, seja em nível de graduação, pós-graduação ou em cursos de formação continuada, a maioria informou que se sente totalmente ou parcialmente preparada para atuar em tal contexto.

Porém, o não recebimento dessa capacitação pode estar refletindo na insegurança para atuar, citada por 20 deles (parcialmente preparado). Por outro lado, esse achado pode indicar, também que, embora não tenham recebido, na educação formal, os conhecimentos teóricos e práticos acerca de sua atuação, parte dos fisioterapeutas deste estudo têm buscado informações de forma independente para se inserirem na proposição e desenvolvimento de ações, tendo em vista que 40,4\% disseram atuar visando à inclusão do alunado em questão.

Nesse caso, pode-se questionar que tipo de atendimento tem sido realizado, em que tem se baseado os profissionais e se essas ações têm sido adequadas para suprir as demandas do contexto educacional inclusivo.

Além da ausência nas diretrizes curriculares do curso, é possível que ainda sejam escassos os Programas de Pós-graduação na área da fisioterapia 
no Brasil que ofereçam, entre as linhas de pesquisa, investigações voltadas para a inclusão escolar, já que os estudos stricto-sensu encontrados no trabatho de Martins e Melo (2010) que tratavam dessa interface eram oriundos de Programas de Pós-graduação em Educação, Educação Especial ou áreas afins.

No contexto em que boa parte das universidades não abordam o tema da inclusão escolar como parte integrante do currículo de fisioterapeutas, portanto, que não fornecem o embasamento teórico e prático para atuação na escola com pessoas com deficiência física, perpetua-se uma visão tecnicista de tratamento, reabilitação e/ou manutenção, concentrando-se os campos de atuação em clínicas e hospitais (BISPO JUNIOR, 2009).

Em pesquisa objetivando identificar alterações nas concepções de deficiência e reabilitação de alunos do primeiro e do último ano de um curso de graduação em Fisioterapia em uma universidade brasileira, Lamônico e Cazeiro (2006) verificaram, ao final do curso, uma maior atenção para com os aspectos técnico-científicos da deficiência em contraposição às suas implicações sociais. Ademais, os dados, referentes ao conceito de reabilitação, evidenciaram quanto à categoria que a reintegração à sociedade revelou um lamentável descaso desses alunos para com o mecanismo de inclusão social das pessoas com deficiência.

É importante pontuar aqui que, no Brasil, a reabilitação institui-se como prática especializada na área da saúde para as pessoas com deficiência física nas décadas de 40 e 50, mas somente na década de 90 é que são emanadas uma série de resoluções importantes do Conselho Federal de Fisioterapia e Terapia Ocupacional (COFFITO), legislações oriundas dos Governos Federal e Estaduais, em especial do Ministério da Saúde com relação à fisioterapia. Pode-se dizer que esse campo de conhecimento passou a caminhar em direção ao campo da saúde em toda sua plenitude, voltando-se, inclusive, a questões relacionadas à educação e promoção de saúde (MARTINS, 2002; BARROS, 2003).

A Resolução n. O8 do COFITTO, de 20 de fevereiro de 1978, que aprova as normas para habilitação ao exercício das profissões de Fisioterapeutas e Terapeutas Ocupacionais, publicada no Diário Oficial da União, considera em seu art. $3^{\circ}$ como exercício profissional do fisioterapeuta, entre outros aspectos 
Atuação de fisioterapeutas na inclusão de alunos com deficiência física no ensino regular

[...] prescrever, ministrar e supervisionar terapia física, que objetive preservar, manter, desenvolver ou restaurar a integridade de órgão, sistema ou função do corpo humano, por meio de: [...] educação ou reeducação neuro-muscular, de locomoção, de regeneração ósteo-articular, de correção de vício postural, de adaptação ao uso de órtese ou prótese e de adaptação dos meios e materiais disponíveis, pessoais ou ambientais, para o desempenho físico do cliente (BRASIL, 1978, s/p.).

É possível notar nessa resolução citada que, mediante o detalhamento das competências do fisioterapeuta, faz-se uma relação com o que é realizado no ambiente escolar, permitindo fazer referência não apenas à deficiência ou impossibilidade da pessoa, mas também ao entendimento de que a deficiência advém da interação do indivíduo com o ambiente (MARTINS, 2002).

Desta forma, é possível fazer uma relação dessa concepção com o modelo da CIF, no qual as abordagens visam não só as mudanças de estrutura e função do corpo, mas também incluem mudanças no desempenho das pessoas com condições de saúde diversas, considerando ainda a influência dos contextos e a possibilidade de modificação dos fatores ambientais.

De fato, no ano de 2009, por meio da Resolução n 370, de 6 de novembro de 2009, o COFFITO recomenda que os fisioterapeutas utilizem a CIF com objetivos diversos, inclusive como ferramenta clínica, considerando que a redução da incapacidade, a promoção e proteção da funcionalidade são preocupações pertinentes dos fisioterapeutas.

Rosenbaum e Stewart (2004) destacam que os aspectos avaliados na criança com deficiência física precisam ser multidimensionais, expandindo o impacto do que é feito para os diferentes níveis de função e estrutura corporal, atividade e participação. Essa ênfase deveria ser dada quanto à determinação da influência dos elementos pessoais e ambientais sobre a saúde e funcionalidade da pessoa.

No entanto, ao realizar uma revisão dos trabalhos publicados em revistas indexadas sobre as formas de uso da CIF em Fisioterapia no Brasil, Araújo (2008) encontrou 18 artigos revelando uma utilização ainda parcial da classificação pelos profissionais, sendo apontadas dificuldades para a sua execução nos serviços.

Além da possibilidade que o fisioterapeuta que trabalha no ambiente clínico com crianças e adolescentes com deficiência física em idade escolar 
possui de desenvolver ações na perspectiva da inclusão, acredita-se que tal responsabilidade deve ser vislumbrada e apropriada pelos fisioterapeutas que trabalham na atenção básica junto aos Núcleos de Apoio à Saúde da Família (NASF).

De fato, com a criação do NASF pela Portaria n 154, de 24 de janeiro de 2008, as atribuições dos profissionais que compõem as equipes, incluindo o fisioterapeuta, perpassam por ações de promoção da saúde e assistência à reabilitação, entre outras, tendo as pessoas com deficiência como uma das prioridades. Inclusive, para o entendimento acerca da deficiência, o documento cita a CIF de 2003.

Esse documento engloba entre o detalhamento das ações de reabilitação:

[...] desenvolver ações integradas aos equipamentos sociais existentes, como escolas, creches, pastorais, entre outros; desenvolver projetos e ações intersetoriais, para a inclusão e a melhoria da qualidade de vida das pessoas com deficiência; orientar e informar as pessoas com deficiência, cuidadores e ACS sobre manuseio, posicionamento, atividades de vida diária, recursos e tecnologias de atenção para o desempenho funcional frente às características específicas de cada indivíduo; realizar encaminhamento e acompanhamento das indicações e concessões de órteses, próteses e atendimentos específicos realizados por outro nível de atenção à saúde; e realizar ações que facilitem a inclusão escolar, no trabalho ou social de pessoas com deficiência (BRASIL, 2008b, s/p.).

Portanto, a referida portaria mencionada acima indica que é dever do fisioterapeuta e demais profissionais de saúde que integram o NASF, o desenvolvimento de ações com vistas à inclusão escolar de pessoas com deficiência, recomendando ainda o enfoque multiprofissional e transdisciplinar, além da execução de ações intersetoriais que integrem outras políticas sociais, a exemplo da educação, esporte, cultura, trabalho e lazer.

A transdisciplinaridade ocorre quando há a reunião de diversos profissionais em um trabalho de equipe e a comunicação não segue um modelo verticalizado, baseado na liderança de um saber sobre outro(s), mas assume uma característica horizontal em que todos os membros, igualmente, compartitham de seus conhecimentos e saberes e tomam decisões relativas ao que está sendo discutido (IRIBARRY, 2003). 
Atuação de fisioterapeutas na inclusão de alunos com deficiência física no ensino regular

Assim, esses profissionais devem atuar, também, como articuladores da rede de serviços, encaminhando, adequadamente, os usuários para os outros níveis de complexidade quando se fizer necessário, objetivando a integralidade do cuidado, a melhoria da qualidade de vida dos indivíduos e sua reinserção social.

Embora exista um carecimento de publicações sobre a atuação do fisioterapeuta na Atenção Básica de Saúde, há algumas similaridades das experiências, a exemplo de ações assistenciais isoladas de pacientes, visitas domiciliares, entre outras (BARBOSA; FERREIRA; FURBINO, 2010; FORMIGA; RIBEIRO, 20121.

No entanto, não foram encontrados estudos que relataram ações que visam promover a inclusão escolar de alunos com deficiência, bem como aqueles que objetivam avaliar se as atividades realizadas por esses profissionais se coadunam com os princípios de atuação, previstos no documento que norteia a atuação nos NASF (FORMIGA; RIBEIRO, 2012).

Apesar de diversos autores concordarem que ainda prevalece a formação tecnicista do profissional fisioterapeuta, centrada nos setores secundário e terciário, a mudança em curso deve ser ampliada no que diz respeito a um processo formativo acerca do processo saúde-doença, a prevenção de doenças, promoção da saúde e qualidade de vida, aproximando a fisioterapia da saúde coletiva, em especial da atenção básica (BISPO JUNIOR, 2009; BARBOSA; FERREIRA; FURBINO, 2010 ; DELAI; WISNIEWSKI, 2011 ; FORMIGA; RIBEIRO, 2012).

Nesse interim, reforça-se a necessidade de também incluir no currículo de fisioterapia no Brasil, conteúdos e práticas para que os profissionais desenvolvam competências e habilidades que thes permitam trabalhar na perspectiva da inclusão escolar de pessoas com deficiência, seja no âmbito da atenção primária ou secundária de saúde.

Ao serem questionados se consideravam o trabalho de uma equipe multidisciplinar efetivo para a inclusão dos alunos com deficiência física na escola, 46 fisioterapeutas do presente estudo consideraram que sim e somente um respondeu que acreditava ser parcialmente efetivo.

Quanto aos recursos humanos, Mendes, Almeida e Toyoda (2011) apontam os modelos de colaboração entre professores, pais e profissionais de 
Francisco Ricardo Lins Vieira de Melo| Neide Maria Gomes de Lucena | Luzia Lívia Oliveira Saraiva

diversas áreas de conhecimento como estratégias bem sucedidas para atender à diversidade no contexto da inclusão.

Entre as formas de colaboração na escola, cita-se a consultoria colaborativa como modelo de suporte baseado no trabalho colaborativo entre profissionais especializados (seja da área da saúde, educação, tecnológica ou assistência sociall) e educadores da escola comum. Assim, esses profissionais atuam como consultores que assistem o professor de sala de aula para maximizar o desenvolvimento educacional dos estudantes (MENDES; ALMEIDA; TOYODA, 20111.

Nesse tipo de atuação, há um intercâmbio de conhecimentos, habilidades e experiências entre segmentos diversos em que os saberes são valorizados numa perspectiva transdisciplinar.

Acredita-se que o modelo de consultoria colaborativa encontre viabilidade para execução tendo como foco o atendimento das necessidades dos alunos com deficiência física no ensino regular, inclusive como forma de intervenção do fisioterapeuta no ambiente escolar.

Nesse contexto de consultoria colaborativa, a presença e participação do fisioterapeuta podem configurar uma estratégia alternativa para a formação continuada de educadores, sendo possível: identificar as dificuldades dos professores; avaliar as limitações ambientais e necessidades do aluno; desenvolver planejamento colaborativo com o professor; indicar/desenvolver adaptações; orientar/treinar recursos humanos /pais, professores, funcionários e alunos); organizar/modificar o ambiente; e avaliar os resultados, a efetividade e aceitação dos alunos e professores, usuários das estratégias e recursos implementados (ALPINO, 2008).

Sendo assim, se reitera a atenção para o processo formativo do fisioterapeuta. É necessário, pois, que os cursos propiciem espaços para que os graduandos desenvolvam habilidades com vistas ao trabalho multiprofissional e transdisciplinar, ampliando a interação entre os saberes e aplicando-os ao contexto das ações integradas de saúde e educação. 


\section{Considerações finais}

Com base no objetivo proposto no presente artigo, qual seja, identificar a atuação do fisioterapeuta no processo de inclusão escolar de alunos com deficiência física, considerando a política de educação especial na perspectiva inclusiva vigente no contexto brasileiro, evidencia-se que, apesar dos avanços em termos legais, a implementação e a discussão do paradigma da educação inclusiva ainda não se efetivaram no currículo de muitos cursos de graduação no Ensino Superior, inclusive nos Cursos de Fisioterapia.

A fala dos participantes da pesquisa, fisioterapeutas com tempo médio de formação acadêmica de 9, 1 anos, evidencia essa leitura ao ser constatada que, apenas, 8 dos 47 participantes, cursaram alguma disciplina específica voltada ao atendimento educacional da pessoa com deficiência física em sua graduação. Tal fato pode ser considerado como uma das variáveis importantes que justifica a não participação desses profissionais $(59,6 \%)$, atuando no processo de inclusão escolar de crianças e adolescentes com deficiência física.

A realidade trazida pelas falas dos fisioterapeutas investigados denuncia uma formação acadêmica mais condizente com o avanço das concepções acerca da deficiência, contemplando aspectos educacionais, funcionais e biopsicossociais, fundamentada no modelo da Classificação Internacional de Funcionalidade, Incapacidade e Saúde - CIF IORGANIZAÇÃO MUNDIAL DE SAÚDE, 2004).

É preciso que, no currículo dos fisioterapeutas, conteúdos acerca da educação das pessoas com deficiência sejam abordados, assim como sejam privilegiadas, durante sua formação, práticas em contextos escolares onde esses profissionais possam adquirir competências e habilidades para o trabalho junto à equipe com vistas à inclusão do aluno com deficiência física. A ausência de informações e experiências práticas sobre essa temática, durante a trajetória acadêmica dos fisioterapeutas participantes do estudo, reflete essa proposição, uma vez que, apenas, 18 fisioterapeutas relataram se sentir preparados para atuar com esse público nas escolas.

Ao aprofundar a discussão do aparato legal que dá suporte à atuação do fisioterapeuta, na inclusão de alunos com deficiência física na escola regular, ressaltam-se a urgência e a necessidade de investigações na área tendo em vista a escassez na literatura nacional. 
Francisco Ricardo Lins Vieira de Melo| Neide Maria Gomes de Lucena | Luzia Lívia Oliveira Saraiva

Ademais, é preciso que as políticas públicas educacionais e de saúde sejam mais bem definidas, estruturadas e articuladas, no que diz respeito aos serviços prestados pelo fisioterapeuta no sistema educacional, tendo em vista a importância da interdisciplinaridade na efetivação de uma escola verdadeiramente inclusiva.

\section{Referências}

ALPINO, Ângela Maria Sirena. Consultoria colaborativa escolar do fisioterapeuta: acessibilidade e participação do aluno com paralisia cerebral em questão. 2008. 192f. Tese (Doutorado em Educação em Especial) - Programa de Pós-Graduação em Educação Especial, Universidade Federal de São Carlos, São Carlos, 2008.

ALPINO, Ângela Maria Sirena; SILVA, Carolina Kruleske da; KARIATSUMARI, Cinthia Tiemi; SOUZA, Jenifer Silva de; OKURO, Renata Tiemi; ARAÚjO, Tatiana Abade Ferreira de. Programa de promoção e apoio a inclusão de crianças com deficiência física: a fisioterapia no contexto da educação inclusiva. In: CONGRESSO BRASILEIRO MULTIDISCIPLINAR DE EDUCAÇÃO ESPECIAL, 4., 2007, Londrina. Anais... Londrina: Universidade Estadual de Londrina 2007. Disponível em: <http://www.uel.br/eventos/congressomultidisciplinar/ pages/arquivos/anais/2007/010.pdf>. Acesso em: 31 maio 2017.

ALPINO, Ângela Maria Sirena. $O$ aluno com paralisia cerebral no ensino regular: ator ou expectador do processo educacional? 2003. 14 If. Dissertação Mestrado em Educação Especial) - Programa de Pós-Graduação em Educação Especial, Universidade Federal de São Carlos, 2003.

ARANHA, Maria Salete Fábio (Org.). Educação inclusiva: a escola. Brasília, DF: MEC/ Secretaria de Educação Especial, 2004. 3 v. Disponível em: <http://portal.mec.gov.br/ seesp/arquivos/pdf/aescola.pdf>. Acesso em: 31 maio 2017.

ARAÚJO, Eduardo Santana de. A classificação internacional de funcionalidade, incapacidade e saúde (CIF) em Fisioterapia: uma revisão bibliográfica. 2008. 117 f. Dissertação (Mestrado em Saúde Pública) - Programa de Pós-Graduação em Saúde Pública, Universidade de São Paulo, 2008. São Paulo, 2008.

ARNS, Ulrika. Por uma fisioterapia que pergunte pela criança: a construção epistemológica e políitica de uma práxis. 2006. 174f. Tese (Doutorado) - Programa de Pós-graduação em Educação, Universidade Federal do Rio Grande do Sul, Porto Alegre, 2006. 
Atuação de fisioterapeutas na inclusão de alunos com deficiência física no ensino regular

BARBOSA, Erika Guerrieri; FERREIRA, Dircilene Leite Santos; FURBINO, Sheila Aparecida Ribeiro. Experiência da fisioterapia no Núcleo de Apoio à Saúde da Família em Governador Valadares, MG. Fisioterapia em Movimento, Curitiba, v. 23, n. 2, p. 323-330, jun. 2010. Disponível em: <http://www.scielo.br/scielo.php?script=sci_arttext\&pid=S0103$-51502010000200015 \&$ lng=en\&nrm=iso >. Acesso em: 1 jun. 2017.

BARROS, Fábio Batalha Monteiro. Autonomia profissional do fisioterapeuta ao longo da história. Revista FisioBrasil, Rio de Janeiro, v. 59, p. 20-31, 2003. Bimensal.

BISPO JUNIOR, José Patrício. Formação em fisioterapia no Brasil: reflexões sobre a expansão do ensino e os modelos de formação. História, Ciências, Saúde-Manguinhos, Rio de Janeiro, v. 16, n. 3, p. 655-668, set. 2009. Disponível: em: <http://www.scielo.br/scielo. php? script=sci_arttext\&pid=SO 104-59702009000300005\&lng=en\&nrm=iso >. Acesso em: 1 jun. 2017.

BRACCIALLI, Lígia Maria Presumido; MANZINI, Eduardo José; REGANHAN, Walkiria Gonçalves. Contribuição de um programa de jogos e brincadeiras adaptados para a estimulação de habilidades motoras em alunos com deficiência física. Temas sobre Desenvolvimento, São Paulo, v. 13, p. 37-46. 2004. Disponível em: http://27reuniao.anped.org.br/gt15/ t154.pdf. Acesso em: 31 maio 2017.

194 BRASIL. Resolução n. 08, de 20 de fevereiro de 1978. Aprova as Normas para habilitação ao exercício das profissões de fisioterapeuta e terapeuta ocupacional e dá outras providências. Diário Oficial [da] República Federativa do Brasil, Brasília, DF, Brasília, n. 216 , p. $6.322 / 32$, nov. 1978 .

BRASIL. Portaria n 1.793, de 27 de dezembro de 1994. Diário Oficial [da] República Federativa do Brasil, Brasília, DF, 27 dez. 1994, Brasília, 1994.

BRASIL. Lei n 9.394, de 20 de dezembro de 1996. Estabelece as diretrizes e bases da educação nacional. Brasília, DF, 1996. Disponível em: <http://www.planalto.gov.br/ccivil_03/leis/19394.htm>. Acesso em: 31 maio 2017.

BRASIL. Ministério da Educação. Secretaria de Educação Especial. Adaptações Curriculares em Ação: desenvolvendo competências para o atendimento às necessidades educacionais de alunos com deficiência física/neuromotora. Brasília: SEESP, 2002.

BRASIL. Ministério da Educação. Secretaria de Educação Especial. Política Nacional de Educação Especial na Perspectiva da Educação Inclusiva. Brasília: SEESP, 2008a. Disponível em: <http://portal.mec.gov.br/arquivos/pdf/politicaeducespecial.pdf>. Acesso em: 31 maio 2017. 
Francisco Ricardo Lins Vieira de Melo| Neide Maria Gomes de Lucena | Luzia Lívia Oliveira Saraiva

BRASIL. Portaria n 154, de 24 de janeiro de 2008. Cria os Núcleos de Apoio à Saúde da Família - NASF. Diário Oficial [da] República Federativa do Brasil, Brasília, DF, 24 jan. 2008, Brasília, 2008b.

BRASIL. Resolução n 370, de 6 de novembro de 2009. Dispõe sobre a adoção da Classificação Internacional de Funcionalidade, Incapacidade e Saúde (CIF) da Organização Mundial de Saúde por Fisioterapeutas e Terapeutas Ocupacionais. Diário Oficial [da] República Federativa do Brasil, Poder Executivo, Brasília, DF, 25 nov. 2009, Seção 1, p. 101.

DELAI, Kéllin Daneluz; WISNIEWSKI, Miriam Salete Wilk. Inserção do fisioterapeuta no Programa Saúde da Família. Ciência \& saúde coletiva, Rio de Janeiro, v. 16, p. 1515-1523, 2011 . (Supl. 1). Disponível em: <http://www.scielo.br/scielo.php? script=sci_ arttext\&pid=S 141 3-81232011000700087\&lng=pt\&nrm=iso>. Acesso em: 1 jun. 2017.

DURCE, Karina; PEREIRA, Priscila Souza; FERREIRA, Cláudia Adriana SantAnna; SOUZA, Brenda Balbino. A atuação da fisioterapia na inclusão de crianças deficientes físicas em escolas regulares: uma revisão de literatura. O Mundo da Saúde, São Paulo, v. 30, n. 1, p. 156-159. 2006.

FERREIRA, Solange Leme. Ingresso, permanência e competência: uma realidade possível para universitários com necessidades educacionais especiais. Revista Brasileira de Educação Especial, Marília, v. 13, n. 1, p. 43-60, abr. 2007. Disponível em: <http://www.scielo. $\mathrm{br} /$ scielo.php? script=sci_arttext\&pid=S $1413-65382007000100004 \&$ lng =en\&nrm=iso $>$. Acesso em: 31 maio 2017.

FORMIGA, Nicéia Fernandes Barbosa; RIBEIRO, Kátia Suely Queiroz Silva. Inserção do fisioterapeuta na atenção básica: uma analogia entre experiências acadêmicas e a proposta dos Núcleos de Apoio à Saúde da Família (NASF). Revista Brasileira de Ciências da Saúde, v. 16, n. 2, p. 113-122. 2012. Disponível em: <http://periodicos.ufpb.br/ojs/index.php/ rbcs/article/download/10639/7300>. Acesso em: 31 maio 2017.

GALLO, Emanuela Cerutti G.; ORSO, Kelen Daiane; FIÓRIO, Franciane Barbieri. Análise da acessibilidade das pessoas com deficiência física nas escolas de Chapecó-SC e o papel do fisioterapeuta no ambiente escolar. $\bigcirc$ Mundo da Saúde, São Paulo, v. 35, n. 2, p. 201-207. 2011. Disponível em: <http://bvsms.saude.gov.br/bvs/artigos/analise_acessibilidade_pessoas_deficiencia_fisica_escolas.pdf>. Acesso em: 31 maio 2017.

GIL, Antônio Carlos. Como elaborar projetos de pesquisa. 5. ed. São Paulo: Atlas, 2010.

IRIBARRY, Isac Nikos. Aproximações sobre a transdisciplinaridade: algumas linhas históricas, fundamentos e princípios aplicados ao trabalho de equipe. Psicologia: Reflexão e Critica, 
Atuação de fisioterapeutas na inclusão de alunos com deficiência física no ensino regular

Porto Alegre, v. 16, n. 3, p. 483-490. 2003. Disponível em: <http://www.scielo.br/ scielo.php?script=sci_arttext\&pid=SO 102-79722003000300007\&lng=en\&nrm=iso > . Acesso em: 1 jun. 2017.

JORQUEIRA NETO, Adriana Cristina; BLASCOVI-ASSIS, Silvana Maria. Contribuições do fisioterapeuta na inclusão escolar de alunos com deficiência sob a perspectiva do brincar. Cadernos de Pós-Graduação em Distúrbios do Desenvolvimento, São Paulo, v. 9, n. 1, p. 76-91. 2009. Disponível em: <http://www.mackenzie.br/fileadmin/Graduacao/CCBS/ Pos-Graduacao/Docs/Cadernos/Caderno_vol_8/2009.2Artigo_5_CONTRIBUli__c_ ES_DO_FISIOTERAPEUTA_NA_INCLUS_ESCOLAR_DE_ALUNOS_COM_DEFICI_CIA_ SOB_A_PERSPECTIVA_DO_BRINCAR.pdf>. Acesso em: 31 maio 2017.

LANDMANN, Luciana Machado; RUZZA, Poliana; CHESANI, Fabíola Hermes. Espaço educacional e a possibilidade de atuação do fisioterapeuta. Ciências \& Cognição, Rio de Janeiro, v. 14, p. 83-91. 2009. Disponível em: <http://www.cienciasecognicao.org/ revista/index.php/cec/article/view/247>. Acesso em: 31 maio 2017.

LOMÔNACO, José Fernando Bitencourt; CAZEIRO, Ana Paula Martins. Concepções de deficiência e reabilitação: um estudo exploratório com graduandos de fisioterapia. Psicologia Escolare Educacional, Campinas, v. 10, n. 1, p. 83-97, jun. 2006. Disponível em <http://pepsic.bvsalud.org/scielo.php? script=sci_arttext\&pid=S 141 3-85572006000100008\&lng=pt \&nrm=iso>. Acesso em: 5 jun. 2017.

MARTINS, Juliana Soccol. Atuação do fisioterapeuta na realidade escolar de crianças com deficiência física: uma perspectiva integradora. 2002. 129f. Dissertação (Mestrado em Distúrbios do Desenvolvimento) - Pós-Graduação em Distúrbios do Desenvolvimento, Universidade Presbiteriana Mackenzie, São Paulo. 2002.

MARTINS, Vanessa Dantas; MELO, Francisco Ricardo Lins Vieira de. Análise de resumos de dissertações e teses relacionadas a fisioterapia e educação inclusiva no contexto brasileiro ( 1999-2008). In: MARTINS, Lúcia de Araújo Ramos; SILVA, Luzia Guacira dos Santos; PIRES, José; PIRES, Gláucia Nascimento da Luz (Org.). Educação e diversidade: saberes e experiências. João Pessoa: Editora Universitária UFPB, 2010.

MELO, Francisco Ricardo Lins Vieira de Melo. Do olhar inquieto ao olhar comprometido: uma experiência de intervenção voltada para atuação com alunos que apresentam paralisia cerebral. 2006. 266f. Tese (Doutorado em Educação) - Programa de Pós-Graduação em Educação, Universidade Federal do Rio Grande do Norte, Natal, 2006.

MELO, Francisco Ricardo Lins Vieira de; FERREIRA, Caline Cristine de Araújo. $\bigcirc$ cuidar do aluno com deficiência física na educação infantil sob a ótica das professoras. Revista Brasileira 
Francisco Ricardo Lins Vieira de Melo| Neide Maria Gomes de Lucena | Luzia Lívia Oliveira Saraiva

de Educação Especial, Marília, v. 15, n. 1, p. 121 1-140, abr. 2009. Disponível em: <http:// www.scielo.br/scielo.php?script=sci_arttext\&pid=S 141 3-65382009000 100009\&lng=en \&nrm=iso>. Acesso em: 1 jun. 2017.

MELO, Francisco Ricardo Lins Vieira de; PEREIRA, Ana Paula Medeiros. Inclusão escolar do aluno com deficiência física: visão dos professores acerca da colaboração do fisioterapeuta. Revista Brasileira de Educação Especial, Marília, v. 19, n. 1, p. 93-106, mar. 2013. Availablefrom<http://www.scielo.br/scielo.php? script=sci_arttext\&pid=S14 13 -65382013000100007\&lng=en\&nrm=iso>. Acesso em: 5 jun. 2017.

MENDES, Eniceia Gonçalves (Org.). Colaboração entre ensino regular e especial: o caminho do desenvolvimento especial. Inclusão e Acessibilidade. Marília: ABPEE; 2006.

MENDES, Eniceia Gonçalves; ALMEIDA, Maria Amélia; TOYODA, Cristina Yoshie. Inclusão escolar pela via da colaboração entre educação especial e educação regular. Educar em Revista, Curitiba, v. 41, p. 81-93, jul./set. 2011 . Disponível em: <http://www.scielo.br/ $\mathrm{pdf} / \mathrm{er} / \mathrm{n} 41 / 06 . \mathrm{pdf}>$. Acesso em: 31 maio 2017.

MORINA, Élido José. A inclusão do aluno com paralisia cerebral: demanda do professor para apoio técnico em fisioterapia. 2013. 63f. Dissertação (Mestrado em Ciências Médicas) - Pós-Graduação da Faculdade de Ciências Médicas, Universidade Estadual de Campinas, Campinas, 2013.

NIEHUES, Janaina Rocha; NIEHUES, Mariane Rocha. Educação Inclusiva de Crianças com Deficiência Física: Importância da Fisioterapia no Ambiente Escolar. Revista Neurociências, São Paulo, v. 22, n. 1, p. 113-120. Jan./mar. 2014. Disponível em: <http://www.revistaneurociencias.com.br/edicoes/2014/2201/2201 revisao/893revisao.pdf>. Acesso em: 31 maio 2017.

NOVAK, lona; MCINTYRE, Sarah; MORGAN, Catherine; CAMPBELL, Lanie; DARK, Leigha; MORTON, Natalie; STUMBLES, Elise; WILSON, Salli-Ann; GOLDSMITH, Shona. A systematic review of interventions for children with cerebral palsy: state of the evidence. Developmental Medicine \& Child Neurology, London, v. 55, n. 10, p. 885-910, out. 2013. Disponível em: <http://onlinelibrary.wiley.com/doi/10.1111/dmcn.12246/fulls. Acesso em: Acesso em: 31 maio 2017 .

OlIVEIRA, Edja Renata Marques de; MELO, Francisco Ricardo Lins Vieira de; ELALI, Gleice Virginia Medeiros de Azambuja. Acessibilidade e participação de estudantes com deficiência física na Universidade Federal do Rio Grande do Norte. Revista Educação em Questão, Natal, v. 33, n. 19, p. 63-87, set./dez. 2008. Disponível em: <https://periodicos.ufrn. br/educacaoemquestao/article/download/3927/3194>. Acesso em: 31 maio 2017. 
Atuação de fisioterapeutas na inclusão de alunos com deficiência física no ensino regular

ORGANIZAÇÃO MUNDIAL DA SAUUDE. Relatório mundial sobre a deficiência. São Paulo: SEDPCD, $2012.334 \mathrm{p}$.

ORGANIZAÇÃO MUNDIAL DE SAÚDE. Classificação Internacional de Funcionalidade, Incapacidade e Saúde. Lisboa: Direcção Geral da Saúde, 2004. 237p.

ROSENBAUM, Peter; STEWART, Debra. The world organization international classification of functioning, disability, and health: a model to guide clinical thinking, practice and research in the field of cerebral palsy. Seminars in Pediatric Neurology, Philadelphia, v. 1 1, n. 1, p. 5-10. 2004.

SANTOS, Marcelli Evans Telles dos; LARA, Simone; FOLMER, Vanderlei. Inclusão escolar: possíveis contribuições da fisioterapia sob a óptica de professoras. Revista Educação Especial, Santa Maria, v. 28, n. 51, p. 67-82, jan./abr. 2015. Disponível em:<https:// periodicos.ufsm.br/educacaoespecial/article/view/5701>. Acesso em: 26 maio 2017.

SARAIVA, Luzia Lívia Oliveira; MELO, Francisco Ricardo Lins Vieira de. Avaliação e participação do fisioterapeuta na prescrição do mobiliário escolar utilizado por alunos com paralisia cerebral em escolas estaduais públicas da rede regular de ensino. Revista Brasileira de Educação Especial, Marília, v. 17, n. 2, p. 245-262, ago. 2011 . Disponível em: <http:// www.scielo.br/scielo.php?script=sci_arttext\&pid=S 141 3-653820 $11000200006 \& \operatorname{lng}=e n$ 198 \&nrm=iso>. Acesso em: 31 maio 2017.

SASSAKI, Romeu Kazumi. Inclusão: o paradigma do século 21. Inclusão: Revista da Educação Especial, Brasília, v. 1, n. 1, p. 19-23, out. 2005.

SILVA, Lívia Joelma Almeida de Lima e; MAZZOTTA, Marcos José da Silveira. Importância da inclusão escolar na reabilitação fisioterapêutica de crianças com paralisia cerebral. Cadernos de Pós-Graduação em Distúrbios do Desenvolvimento, São Paulo, v. 9, n. 1, p. 9-32, 2009.

TAGLIARI, Carina; TRÊS, Francesca; OLIVEIRA, Sheila Gemelli. Análise da acessibilidade dos portadores de deficiência física nas escolas da rede pública de passo fundo e o papel do fisioterapeuta no ambiente escolar. Revista neurociências, São Paulo, v. 14, n. 1, p. 10-14, jan./mar. 2006. Disponível em: <https://www.revistaneurociencias.com.br/ edicoes/2006/RN\%2014\%2001/Pages\%20from\%2ORN\%2014\%2001-2.pdf>. Acesso em: 31 maio 2017. 
Prof. Dr. Francisco Ricardo Lins Vieira de Melo Universidade Federal do Rio Grande do Norte Departamento de Fisioterapia Programa de Pós-graduação em Educação Grupo de Pesquisa: Educação e Inclusão em Contextos Educacionais E-mail | ricardolins67@gmail.com

Profa. Dra. Neide Maria Gomes de Lucena Universidade Federal da Paraíba Departamento de Fisioterapia Programa de Pós-graduação em Engenharia de Produção Grupo de pesquisa: Fisioterapia, Intervenção e Qualidade de Vida E-mail | neidemariaglucena@hotmail.com

Profa. Ms. Luzia Lívia Oliveira Saraiva Universidade Federal do Rio Grande do Norte

Faculdade de Ciências da Saúde do Trairi Grupo de Pesquisa: Ambiente, participação e qualidade de vida de crianças e adolescentes com deficiência física E-mail | liviaosaraiva@hotmail.com

Recebido 19 jun. 2017 Aceito 31 jul. 2017 